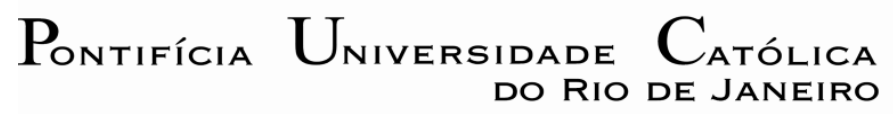

Ana Carolina Barbosa Freire

Previsão de Câmbio e Preços de Commodities: uma análise empírica do caso brasileiro

Dissertação de Mestrado

Dissertação apresentada como requisito parcial para obtenção do título de Mestre pelo Programa de PósGraduação em Economia da PUC-Rio.

Orientador: Prof. Afonso Sant'Anna Bevilaqua Co-Orientador: Prof. Marcelo Cunha Medeiros 


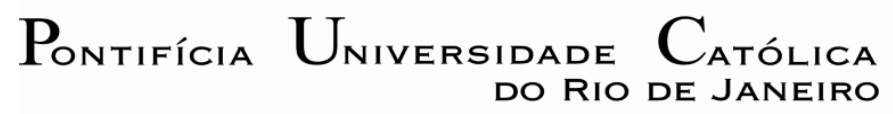

DO RIO DE JANEIRO

Ana Carolina Barbosa Freire

\section{Previsão de Câmbio e Preços de Commodities: uma análise empírica do caso brasileiro}

Dissertação apresentada como requisito parcial para obtenção do título de Mestre pelo Programa de PósGraduação em Economia da PUC-Rio. Aprovada pela Comissão Examinadora abaixo assinada.

Prof. Afonso Sant'Anna Bevilaqua
Orientador
Departamento de Economia - PUC-Rio

Prof. Marcelo Cunha Medeiros Co-Orientador Departamento de Economia - PUC-Rio

Prof. Eduardo Henrique de Mello Motta Loyo Departamento de Economia - PUC-Rio/UBS

Prof. Alexandre Schwartsman Santander

Prof. Nizar Messari Coordenador Setorial do Centro de Ciências Sociais - PUC-Rio

Rio de Janeiro, 31 de março de 2009 
Todos os direitos reservados. É proibida a reprodução total ou parcial do trabalho sem autorização da universidade, da autora e do orientador.

\section{Ana Carolina Barbosa Freire}

Graduou-se em Economia pela PUC-Rio em 2006.

Ficha Catalográfica

Freire, Ana Carolina Barbosa

Previsão de Câmbio e Preços de Commodities: uma análise empírica do caso brasileiro / Ana Carolina Barbosa Freire; orientador: Afonso Sant'Anna Bevilaqua; coorientador: Marcelo Cunha Medeiros. - Rio de Janeiro: PUC, Departamento de Economia, 2009.

113 f. : il. (color.) ; $30 \mathrm{~cm}$

Dissertação (Mestrado em Economia) - Pontifícia Universidade Católica do Rio de Janeiro, Rio de Janeiro, 2009.

Inclui bibliografia.

1.Economia - Teses. 2. Commodity currencies. 3. Câmbio real. 4. Câmbio nominal. 5. Preços de commodities. 6. Previsão. 7. Cointegração. I. Bevilaqua, Afonso Sant'Anna. II. Medeiros, Marcelo Cunha. III. Pontifícia Universidade Católica do Rio de Janeiro. Departamento de Economia. IV. Título. 


\section{Agradecimentos}

Aos meus orientadores, Afonso Bevilaqua e Marcelo Medeiros, pela atenção especial, pelos conselhos que tanto contribuíram para esta pesquisa, e pelas conversas nas horas importantes, que me ajudaram a finalizar o meu trabalho.

Aos integrantes da minha Banca, Eduardo Loyo e Alexandre Schwartsman, pelos comentários e sugestões que certamente melhoraram a qualidade da minha pesquisa.

Aos professores Márcio Garcia, Rogério Werneck e Fabio Giambiagi não apenas pela contribuição acadêmica que me deram ao longo dos últimos anos, mas também pelos excelentes conselhos de vida aos quais serei eternamente grata.

À toda a minha família, especialmente minha mãe, Fátima, meu pai, Bruno, e minha irmã Beatriz pelo apoio no âmbito pessoal, pela educação e pelo incentivo a concluir mais esta etapa da minha vida.

Ao Davi, pessoa muito especial, que me ajudou colaborando e me entendendo nos momentos mais difíceis, estando sempre ao meu lado.

Ao Governo brasileiro pela bolsa de estudos oferecida através do CNPq. 


\section{Resumo}

Freire, Ana Carolina Barbosa; Bevilaqua, Afonso Sant'Anna; Medeiros, Marcelo Cunha. Previsão de Câmbio e Preços de Commodities: uma análise empírica do caso brasileiro. Rio de Janeiro, 2009. 113p. Dissertação de Mestrado - Departamento de Economia, Pontifícia Universidade Católica do Rio de Janeiro.

A literatura teórica sobre taxas de câmbio apresenta uma série de resultados de difícil respaldo empírico como o forecasting puzzle da taxa de câmbio. Ao realizarmos previsões dentro da amostra e fora da amostra para as taxas de câmbio, nominal e real, e para o índice de preços de commodities do Brasil, encontramos evidências empíricas que comprovam algumas das explicações para este puzzle. Basicamente, os resultados dentro e fora da amostra apontam que o câmbio nominal apresenta um forte componente forward looking, o que poderia explicar o fracasso de muitos modelos em prever esta variável. Os valores passados do câmbio nominal conseguem gerar previsões para preços de commodities substancialmente melhores que a de um passeio aleatório, tanto no curto quanto no longo prazo, embora a relação reversa não se verifique. Uma análise comparando as previsões de nosso modelo a um modelo autorregressivo univariado mostra que esta evidência é mais fraca do que constatamos inicialmente. Para a taxa de câmbio real estendemos a análise feita na literatura de commodity currencies para medir o poder preditivo dos modelos utilizados. Considerando o exercício dentro da amostra, os preços de commodities contribuem significativamente para as previsões da taxa de câmbio, mas a causalidade no sentido contrário também ocorre. Já para as previsões fora da amostra, o modelo de correção de erros não conseguiu superar o passeio aleatório para nenhuma variável, nem mesmo no longo prazo. Os resultados são robustos à presença de quebras estruturais.

\section{Palavras-chave}

Commodity currencies, câmbio real, câmbio nominal, preços de commodities, previsão, cointegração. 


\section{Abstract}

Freire, Ana Carolina Barbosa; Bevilaqua, Afonso Sant'Anna (Advisor); Medeiros, Marcelo Cunha (Advisor). Exchange Rates and Commodity Prices Forecasts: an empirical analysis of the Brazilian case. Rio de Janeiro, 2009. 113p. MSc. Dissertation - Departamento de Economia, Pontifícia Universidade Católica do Rio de Janeiro.

In the theoretical literature on exchange rates we find many results with little empirical support. This paper seeks to contribute to a better understanding on one of the main issues that were not fully answered: the forecasting puzzle on exchange rates. By analyzing in-sample and out-of-sample forecasts for nominal and real exchange rates and the commodity price index of Brazil, we find empirical evidence on some of the explanations for this puzzle. The results insample and out-of-sample indicate that the Brazilian nominal exchange rate has an important forward looking component, which could explain the failure of many models to predict this variable. Past values of nominal exchange rate forecasts commodity prices substantially better than a random walk, both in short and long term horizons, while the reverse relation does not hold. An additional analysis in which we compare our model with a univariate autoregressive one suggests that the evidences may not be as robust as we thought at first. For the real exchange rate we extend the analysis made in the literature on commodity currencies to measure the predictive power of these models. Considering the in-sample exercise, commodity prices play an important role in predicting the Brazilian real exchange rate. However, there is also Granger causality in the opposite direction. Regarding the out-of-sample forecasts, our error correction model could not overcome the random walk model. Our results are robust to structural breaks.

\section{Keywords}

Commodity currencies, exchange rates, commodity prices, forecast, cointegration. 


\section{Sumário}

1 Introdução

2 Revisão da Literatura

18

2.1. Taxa de Câmbio Nominal: valor presente dos fundamentos econômicos 18

2.2. A taxa de câmbio real e a literatura sobre commodity currencies

3 Análise do caso brasileiro e descrição dos dados 35

3.1. Análise do caso brasileiro 35

3.2. Descrição dos dados

4 Mecanismo gerador dos dados, quebras estruturais e cointegração 49

4.1. Mecanismo gerador de dados 49

4.2. Cointegração e quebras estruturais

5 Teste de causalidade de Granger e previsões out-of-sample 56

5.1. Previsibilidade In-Sample 56

5.2. Previsibilidade Out-of-Sample 64

6 Análises Adicionais 79

6.1. Diferentes Taxas de Câmbio Nominais 79

6.2. Índice de Preços de Commodities Mundial 82

7 Conclusão

85

8 Referências

9 Apêndice Técnico A 


\section{Lista de figuras}

Figura 1 - Câmbio vs. Preços de Commodities 36

Figura 2 - Valor exportado: Total e Commodities 37

Figura 3 - Comparação dos índices de preço PCCl vs. PCCL 42

Figura 4 - Comparação dos índices mistos deflacionados 47

Figura 5 - PCCI - Índices Encadeados 93

Figura 6 - PCCI - Índices Diretos 93

Figura 7 - PCCI - Índices Mistos 94

Figura 8 - PCCL - Índices Encadeados 95

Figura 9 - PCCL - Índices Diretos $\quad 95$

Figura 10 - PCCL - Índices Mistos 96 


\section{Lista de tabelas}

Tabela 1. Estatísticas brasileiras 36

Tabela 2. Testes de Raiz Unitária 50

Tabela 3. Teste de cointegração Engle-Granger 52

Tabela 4. Testes de Andrews - câmbio nominal 53

Tabela 5. Testes de Andrews - câmbio real 53

Tabela 6. Testes de Gregory e Hansen - cointegração com quebra estrutural $\quad 54$

Tabela 7. Testes de Andrews - variáveis em primeira diferença $\quad 57$

Tabela 8. Testes de causalidade de Granger - câmbio nominal 57

Tabela 8b. Teste de causalidade de Granger - sem testar o intercepto 59

Tabela 9. Teste de Causalidade de Granger - F1 60

Tabela 10. Teste de Causalidade de Granger - F2 61

Tabela 11. Teste de Causalidade de Granger - curto prazo - F1 62

Tabela 12. Teste de Causalidade de Granger - curto prazo - F2 62

Tabela 13. Teste de Causalidade de Granger - longo prazo - F1 63

Tabela 14. Teste de Causalidade de Granger - longo prazo - F2 63

Tabela 15. Previsão de câmbio nominal usando o índice PCCL 67

Tabela 16. Previsão de câmbio nominal usando o índice PCCI 67

Tabela 17. Previsão do índice PCCL usando câmbio nominal 69

Tabela 18. Previsão do índice PCCl usando câmbio nominal 69

Tabela 19. Previsão de câmbio nominal usando o índice PCCL 71

Tabela 20. Previsão de câmbio nominal usando o índice PCCl 72

Tabela 21. Previsão do índice PCCL usando câmbio nominal 72

Tabela 22. Previsão do índice PCCl usando câmbio nominal 73

Tabela 23. Previsão da taxa de câmbio real usando o índice PCCL-CPI 74

Tabela 24. Previsão da taxa de câmbio real usando o índice PCCL-AE $\quad 75$

Tabela 25. Previsão da taxa de câmbio real usando o índice PCCI-CPI 
Tabela 26. Previsão da taxa de câmbio real usando o índice PCCI-AE

Tabela 27. Previsão do índice PCCL-CPI usando a taxa de câmbio real

Tabela 28. Previsão do índice PCCL-AE usando a taxa de câmbio real

Tabela 29. Previsão do índice PCCI-CPI usando a taxa de câmbio real

Tabela 30. Previsão do índice PCCI-AE usando a taxa de câmbio real

Tabela 31. Teste de Causalidade de Granger - Euro 80

Tabela 32. Teste de Causalidade de Granger - Libra 80

Tabela 33. Teste de Causalidade de Granger - lene 81

Tabela 34. Teste de Causalidade de Granger - Variáveis Instrumentais 83

Tabela 35. Teste de Causalidade de Granger - VI - F1 83

Tabela 36. Teste de Causalidade de Granger - VI - F2 83

Tabela 37 Peso das commodities na pauta de exportações brasileiras 91 Tabela 19. Previsão da taxa de câmbio real usando o índice PCCL-CPI - complemento 98

Tabela 20. Previsão da taxa de câmbio real usando o índice PCCL-AE - complemento 98

Tabela 21. Previsão da taxa de câmbio real usando o índice PCCI-CPI - complemento

Tabela 22. Previsão da taxa de câmbio real usando o índice PCCI-AE - complemento

Tabela 23. Previsão do índice PCCL-CPI usando a taxa de câmbio real - complemento

Tabela 24. Previsão do índice PCCL-AE usando a taxa de câmbio real - complemento

Tabela 25. Previsão do índice PCCI-CPI usando a taxa de câmbio real - complemento

Tabela 26. Previsão do índice PCCI-AE usando a taxa de 
câmbio real - complemento

Tabela 38. Previsão do câmbio nominal usando índice PCCL - EURO 101

Tabela 39. Previsão do câmbio nominal usando índice PCCI - EURO 102

Tabela 40. Previsão do índice PCCL usando câmbio nominal - EURO 103

Tabela 41. Previsão do índice PCCl usando câmbio nominal - EURO 103

Tabela 42. Previsão do câmbio nominal usando índice PCCL - LIBRA 104

Tabela 43. Previsão do câmbio nominal usando índice PCCI - LIBRA 105

Tabela 44. Previsão do índice PCCL usando câmbio nominal - LIBRA 105

Tabela 45. Previsão do índice PCCI usando câmbio nominal - LIBRA 106

Tabela 46. Previsão do câmbio nominal usando índice PCCL - IENE 107

Tabela 47. Previsão do câmbio nominal usando índice PCCI - IENE 107

Tabela 48. Previsão do índice PCCL usando câmbio nominal - IENE 108

Tabela 49. Previsão do índice PCCI usando câmbio nominal - IENE 109

Tabela 50. Previsão do câmbio nominal usando índice PCCI - VI 109

Tabela 51. Previsão do índice PCCI - VI usando câmbio nominal 110

Tabela 52. Previsão do câmbio nominal usando índice PCCI

$-\mathrm{VI}-\mathrm{CPI}$

Tabela 53. Previsão do câmbio nominal usando índice PCCl

$-\mathrm{VI}-\mathrm{AE}$

Tabela 54. Previsão do índice PCCI - VI - CPI usando câmbio

nominal

Tabela 55. Previsão do índice PCCI - VI - AE usando câmbio nominal 